\title{
HIMENÓPTEROS PARASITÓIDES (INSECTA, HYMENOPTERA) EM ÁREA AGRÍCOLA DE RIO CLARO, SP, BRASIL
}

\section{Souza ${ }^{1}$, S.M.P. Braga ${ }^{2}$, M.J.O. Campos ${ }^{2}$}

${ }^{1}$ Universidade São Francisco, Rua São Francisco de Assis, 218, CEP 12916-900, Bragança Paulista, SP, Brasil. E-mail: luceli.souza@saofrancisco.edu.br

\section{RESUMO}

\begin{abstract}
Entre maio de 2003 ejunho de 2004 foram coletados, com armadilha de Moericke, no Município de Rio Claro (22 $\left.20^{\prime} \mathrm{S}, 47^{\circ} 32^{\prime} \mathrm{O}\right)$, SP, Brasil, 5.308 indivíduos de himenópteros parasitóides pertencentes a 8 superfamíliase 22 famílias. As famílias Mymaridae, Encyrtidae,ScelionidaeePlatygastridae foram as mais comuns, com freqüência relativa de 30,88\%,19,05\%,14,96\% e 6,69\%, respectivamente, com relação ao total de parasitóides coletados. As demais 18 famílias foram coletadas em freqüência menor do que $5 \%$.
\end{abstract}

PALAVRAS-CHAVE: Agroecossistema, Hymenoptera, parasitóides, levantamento, armadilha Moericke.

\section{ABSTRACT}

PARASITOID HYMENOPTERA (INSECTA) COLLECTED IN AN AGRICULTURAL AREA IN RIO CLARO, SP, BRAZIL. From May 2003 to June 2004, collections were made using the Moericke trap placed in an agricultural area in Rio Claro $\left(22^{\circ} 20^{\prime} \mathrm{S}, 47^{\circ} 32^{\prime} \mathrm{O}\right)$, SP, Brazil, resulting in the capture of a total of 5,308 specimens of parasitoids, belonging to 8 superfamilies and 22 families. Mymaridae, Encyrtidae, Scelionidae and Platygastridae were the most common families, being responsible for $30.88 \%, 19.05 \%, 14.96 \%$ and $6.69 \%$ of the total number of parasitoids collected, respectively. Another 18 families were present with relative frequency lower than $5 \%$.

KEY WORDS: Agroecosystem, Hymenoptera, parasitoids, survey, Moericke trap.

\section{INTRODUÇÃO}

Os parasitóides representam o grupo mais rico de espécies da ordem Hymenoptera: são comuns e abundantes em todos os ecossistemas terrestres, desenvolvem-se como parasitóides (endoparasitóides ou ectoparasitóides) de muitos artrópodes, especialmente insetos (QUICKE, 1997), constituindo-seemimportante elemento de controle das populações de outros insetos devido a sua habilidade em responder à densidade das populações de seus hospedeiros (LA SALLE, 1993), e muito utilizados em programas de controle biológico (PARra et al., 2002).

Existe uma vasta literatura sobre os himenópteros parasitóides abordando principalmente taxonomia, biologia e o uso em controle biológico, mas poucos trabalhos sobre a composição faunística. No Brasil, em área de Mata Atlântica, Azevedo \& SAntos (2000) fizeram o levantamento em Cariacica (ES), Azevedo et al. (2002) em Vitória
(ES), Perioto \& Lara (2003) em Ubatuba (SP), Perioto et al. (2005) em Iguape (SP).

Em área de Cerrado foram feitos alguns trabalhos em diferentes regiões do país, como, por exemplo, MARCHIORI et al. (2001), em Itumbiara (GO), MARCHIORI et al. (2003) em Araporã (MG) e Itumbiara (GO), SILVA et al. (2003), em Lavras (MG), Perioto (1991) e BrAGA (2002), em São Carlos (SP).

Scatolini \&Penteado-Dias (2003), Cirelli\& Penteado-Dias (2003) e Restello \& Penteado-Dias (2006) fizeram levantamentos de Braconidae em diferentes regiões do Brasil.

Pouco se conhece a respeito da composição faunística do grupo em agroecossistemas, exceto pelos trabalhos de Perioto et al. (2002a), Perioto et al. (2002b) e PERIOTO et al. (2004) em culturas de algodão, soja e café, respectivamente, na região de Ribeirão Preto, SP. Na região de Rio Claro, não se tem registro de nenhum levantamento o que justifica a realização deste estudo, que teve por objetivo

${ }^{2}$ Universdade Estadual Paulista, Departamento de Ecologia, Rio Claro, SP, Brasil. 
conhecer a fauna de himenópteros parasitóides em uma área agrícola.

\section{MATERIAL E MÉTODOS}

O levantamento foi realizado entre maio de 2003 a junho de 2004 em uma área de 24,2 ha localizada no Município de Rio Claro, SP, Brasil nesse período, foi realizado o cultivo de sorgo, milho, feijão e trigo, em sistema de rodízio, em plantio direto. As coletas foram realizadas quinzenalmente, utilizando-se 17 armadilhas do tipo Moericke, preenchidas com água e gotas de detergente, colocadas diretamente sobre o solo, distan- tes entre si por $100 \mathrm{~m}$, e expostas por $36 \mathrm{~h}$ no campo. Os himenópteros parasitóides coletados foram mantidos em álcool a $70 \%$ e identificados ao nível de família com auxílio de chaves de identificação de Goulet \& Huber (1993).

O material coletado foi depositado na coleção de Hymenoptera do Centro de Estudos de Insetos Sociais (CEIS) da UNESP de Rio Claro, SP.

\section{RESULTADOS E DISCUSSÃO}

Foram coletados 5.308 himenópteros parasitóides pertencentes a 22 famílias distribuídas em 8 superfamílias (Tabela 1).

Tabela 1 - Número de indivíduos nas diferentes famílias de himenópteros parasitóides coletados em área agrícola no Município de Rio Claro, SP, com a utilização de armadilha de Moericke.

\begin{tabular}{|c|c|c|c|c|}
\hline Superfamília & Família & Total de indivíduoscoletados & FR & FRS \\
\hline \multirow[t]{3}{*}{ Ceraphronoidea } & & 240 & 4.52 & \\
\hline & Ceraphronidae & 221 & 4.16 & 92.08 \\
\hline & Megaspilidae & 19 & 0.36 & 7.92 \\
\hline \multirow[t]{13}{*}{ Chalcidoidea } & & 3.189 & 60.08 & \\
\hline & Eulophidae & 224 & 4.22 & 7.02 \\
\hline & Mymaridae & 1.639 & 30.88 & 51.40 \\
\hline & Pteromalidae & 117 & 2.20 & 3.67 \\
\hline & Encyrtidae & 1.011 & 19.05 & 31.70 \\
\hline & Chalcididae & 72 & 1.36 & 2.26 \\
\hline & Eurytomidae & 28 & 0.53 & 0.88 \\
\hline & Trichogrammatidae & 30 & 0.57 & 0.94 \\
\hline & Eupelmidae & 5 & 0.09 & 0.16 \\
\hline & Aphelinidae & 41 & 0.77 & 1.29 \\
\hline & Torymidae & 15 & 0.28 & 0.47 \\
\hline & Eucharitidae & 1 & 0.02 & 0.03 \\
\hline & Elasmidae & 6 & 0.11 & 0.19 \\
\hline \multirow[t]{2}{*}{ Chrysidoidea } & & 102 & 1.92 & \\
\hline & Bethylidae & 102 & 1.92 & 100.00 \\
\hline \multirow[t]{2}{*}{ Cynipoidea } & & 160 & 3.01 & \\
\hline & Eucoelidae & 160 & 3.01 & 100.00 \\
\hline \multirow[t]{2}{*}{ Evanioidea } & & 15 & 0.28 & \\
\hline & Evaniidae & 15 & 0.28 & 100.00 \\
\hline \multirow[t]{3}{*}{ Ichneumonoidea } & & 217 & 4.09 & \\
\hline & Ichneumonidae & 142 & 2.68 & 65.44 \\
\hline & Braconidae & 75 & 1.41 & 34.56 \\
\hline \multirow[t]{3}{*}{ Platygastroidea } & & 1.149 & 21.65 & \\
\hline & Scelionidae & 794 & 14.96 & 69.10 \\
\hline & Platygastridae & 355 & 6.69 & 30.90 \\
\hline \multirow[t]{2}{*}{ Proctotrupoidea } & & 236 & 4.45 & \\
\hline & Diapriidae & 236 & 4.45 & 100.00 \\
\hline Total parasitóides & & 5.308 & & \\
\hline
\end{tabular}

FR = Freqüência relativa das superfamílias e famílias de himenópteros parasitóides em relação ao total de himenópteros parasitóides coletados.

FRS = Freqüência relativa de himenópteros parasitóides coletados em relação ao total de himenópteros parasitóides da superfamília a que pertencem. 
As superfamílias mais coletadas neste levantamento foram Chalcidoidea e Platygastroidea com $60,08 \%$ e $21,65 \%$, respectivamente, representando $81,73 \%$ do total coletado.

As famílias mais abundantes foram Mymaridae $(30,88 \%)$, Encyrtidae (19,05\%) (Chalcidoidea), Scelionidae $(14,96 \%)$ e Platygastridae $(6,69 \%)$ (Platygastroidea). As dezoito famílias restantes apresentaram frequiências relativas, em relação ao total de parasitóides coletados, inferiores a $5 \%$.

Chalcidoidea é rica em número de espécies e utiliza uma grande diversidade de hospedeiros, sendo 12 ordens de Insecta, 2 ordens de Arachnida (Aranae e Acari) e1 família deNematoda (Anguinidae) (Grisson, 1993). Neste estudo, esta superfamília foi representada por 12 famílias. As famílias Mymaridae (51,40\%), Encyrtidae (31,70\%) e Eulophidae (7,02\%) apareceram com maior freqüência relativa dentro da superfamília, e as demais famílias apresentaram freqüência relativa menor do que $4 \%$.

Os membros da família Mymaridae são espécies parasitóides de ovos de insetos, preferencialmente Homoptera, Hemiptera, Coleoptera e Diptera, depositados em locais escondidos tais como tecidos de plantas, debaixo de casca de árvores e em cavidades do solo. No mês de outubro/ 2003 foram coletados 1.087 indivíduos desta família sendo que, no campo, iniciava-se o plantio de milho e o solo continha o restante da colheita de feijão com muitas larvas de Coleoptera. Nas armadilhas, muitos representantes de Diptera foram coletados, sugerindo uma associação com os hospedeiros da plantação de milho e feijão cultivados nessa propriedade.

Encyrtidae é uma das mais importantes famílias deChalcidoidea utilizadas em programas decontrole biológico, cujas espécies são endoparasitóides de ovos ou larvas de Coleoptera, Diptera, Lepidoptera, Hymenoptera, Neuroptera, Orthoptera, Hemiptera, Homoptera e Arachnida (Goulet \& Huber, 1993). Esses grupos de hospedeiros foram coletados nesta área de estudo nas armadilhas de Moericke, sugerindo também uma associação desses parasitóides com o substrato e seus hospedeiros.
Platygastroidea é a terceira maior superfamília, depois de Ichneumonoidea e Chalcidoidea, e parasitam exclusivamente ovos deinsetos, principalmenteDiptera, Coleoptera, Homoptera e Neuroptera (MASNER, 1993). Neste estudo foi representada pelas duas famílias: Scelionidaequeapareceu com freqüência relativa dentro da superfamília de $69,10 \%$ e Platygastridae com $30,90 \%$.

Scelionidaeé um grupo importante no controle de pragas agrícolas, por atuarem como endoparasitóides solitários de ovos de insetos e aranhas (MASNER,1993).

Amaiorfreqüênciarelativadestasduassuperfamílias pode refletir o fato de serem as mais numerosas em espécies e que utilizam uma ampla diversidade de hospedeiros. Ichneumonoidea, quetambémémuitorica emespécies, nestaárea deestudofoi poucorepresentada (4,09\%). Segundo SHAPIRO \& PiCKERING (2000), em um estudo sobre a estruturação da comunidade da família Ichneumonidae em florestas no Panamá e Costa Rica, observaram que a umidade foi um fator importante na estruturação da comunidade e, provavelmente, a freqüência encontrada neste estudo reflita esta pressão ambiental exercida sobreo grupoem função da dinâmica do plantio nesta área agrícola. A família Braconidae tem sido utilizada como indicador do grau de preservação de ecossistemas (ver citações em Restello \& PenteADO-DIAS, 2006), ea baixafreqüêncianestaárea deestudo provavelmente também reflita a pressão exercida pela dinâmica de plantio sobre este grupo.

As demais superfamílias encontradas apresentaram frequeiencia relativa menor do que $5 \%$, sendo Ceraphronoidea $(4,52 \%)$, Proctotrupoidea $(4,45 \%)$, Ichneumonoidea $(4,09 \%)$, Cynipoidea $(3,01)$, Chrysidoidea (1,92\%) e Evanioidea (0,28\%).

As superfamílias de parasitóides encontradas na área de estudo como as mais representativas, também foram as de maior freqüência encontradas em outras áreas agrícolas (Tabela 2).

Chalcidoidea e Platygastroidea também foram as mais coletadas em cultura decafé $(48,7 \%$ e $17,3 \%)$ eem cultura de soja (44,31\% e 43,51\%) (Ревіотоet al., 2004; Perioto et al., 2002b). Na cultura de algodão Chalcidoidea representou $84,37 \%$ do total coletado e Platygastroidea, 7,06\% (Ревіото et al., 2002a).

Tabela 2 - Dados comparativos de himenópteros parasitóides coletados em culturas agrícolas utilizando armadilha Moericke.

\begin{tabular}{|c|c|c|c|c|c|c|}
\hline Cultura agrícola & $\begin{array}{c}\mathrm{N}^{\circ} \text {. total } \\
\text { exemplares }\end{array}$ & $\begin{array}{c}\mathrm{N}^{\circ} \text {. de } \\
\text { superfamílias }\end{array}$ & $\begin{array}{c}\mathrm{N}^{\circ} \text {. de } \\
\text { famílias }\end{array}$ & $\begin{array}{c}\mathrm{N}^{\circ} \text {. de } \\
\text { armadilhas }\end{array}$ & Período de coleta & Fonte \\
\hline Algodão & 16.166 & 8 & 22 & 84 & $23 / 2$ a $3 / 4 / 2001$ & Perioto et al. (2002a) \\
\hline Soja & 4.969 & 7 & 15 & 84 & $24 / 3$ a $7 / 4 / 2000$ & РеRiOto et al. (2002b) \\
\hline Café & 5.228 & 8 & 21 & 84 & $\begin{array}{l}10 / 12 / 2001 \text { a } \\
3 / 1 / 2002\end{array}$ & Perioto et al. (2004) \\
\hline $\begin{array}{l}\text { Grãos (milho, sorgo, } \\
\text { feijão, trigo) }\end{array}$ & 5.308 & 8 & 22 & 17 & $\begin{array}{l}5 / 5 / 2003 \text { a } \\
21 / 6 / 2004\end{array}$ & Este levantamento \\
\hline
\end{tabular}


Ocorreu predominância da família Encyrtidae em todos os levantamentos realizados em culturas agrícolas; a frequêencia em relação à superfamília que pertencem (FRS) foi de 53,19\% em algodão, 49,1\% em café, 43,82\% em soja (РеRiotoet al., 2002a; Ревіото et al., 2002b; Perioto et al., 2004) e 31,70\% neste levantamento.

A família Mymaridae (Chalcidoidea) apresentou neste levantamento, freqüência em relação à superfamília (FRS) de 51,40\%, maior que a obtida por Perioto et al. (2002a), Perioto et al. (2002b) e Perioto et al. (2004), sendo 16,89\% em algodão, 22,3\% em café e $7,45 \%$ em soja. A maior freqüência nesta área de estudo pode ter sido influenciada pela coleta de 1.087 indivíduos no mês de outubro de 2003, refletindouma maior disponibilidade de hospedeiros nesta área em função do tipo de cultivo e do plantio direto.

As semelhanças nas freqüências relativas de famílias e superfamílias na composição faunística de himenópteros parasitóides nestes diferentes cultivos refletem a disponibilidade de hospedeiros encontrados nestes ambientes e substratos.

\section{REFERÊNCIAS}

AzEvEDO, C.O.;S ANTos, H.S. Perfil da fauna dehimenópteros parasitóides (Insecta, Hymenoptera) em umaárea de Mata Atlântica da Reserva Biológica de Duas Bocas, Cariacica, ES, Brasil. Boletim do Museu de Biologia Mello Leitão (N. Ser.), v.11/12, p.117-126, 2000.

Azevedo, C.O.; Kawada, R.; Tavares, M.T.; Perioto, N.W. Perfil da fauna dehimenópteros parasitóides (Insecta, Hymenoptera) em uma área de Mata Atlântica do Parque Estadual da Fonte Grande, Vitória, ES, Brasil. Revista Brasileira de Entomologia, v.46, n.2, p.133-137, 2002.

Braga, S.M.P. Estudo da biodiversidade dos Braconidae (Hymenoptera, Ichneumonoidea) em três ecossistemas da Universidade Federal de São Carlos, São Carlos, SP. 2002. 198p. Tese (Doutorado em Ecologia e Recursos Naturais) - Centro de Ciências Biológicas e da Saúde, Universidade Federal de São Carlos, São Carlos, 2002.

Cirelli, K.R.N.; Penteado-Dias, A.M. Análise da riqueza da fauna de Braconidae (Hymenoptera, Ichneumonoidea) em remanescentes naturais da Área de Proteção Ambiental (APA) de Descalvado, SP. Revista Brasileira de Entomologia, v.47, n.1, p.89-98, 2003.

Gauld, I.D.; Bolton, B. The Hymenoptera. Oxford: Oxford University Press, 1988. 332p.

Gibson, G.A.P. Superfamilies Mymarommatoidea and Chalcidoidea. In: Goulet, H.; Huber, J.T. (Eds.). Hymenoptera of the world: an identification guide to families. Ontario: Agriculture Canada Publiction, 1993. 668p. Chapter 16.

Goulet, H.; Huber, J.T. Hymenoptera of the world: an identification guide to families. Ontario: Agriculture Canada Publication, 1993. 668p.
LASALLE, J. Parasitic hymenoptera, biological control and biodiversisty. In: LaSAlle, J.; Gauld, I.D. (Eds.) Hymenoptera and Biodiversity. Wallingford (UK): C.A.B. International, 1993. 348p. Chap. 8.

Marchiori, C.H.; Silva, C.G.; Caldas, E.R.; Almeida, K.G.S.; Carvalho, S.A.; Penteado-Dias, A.M.; Diaz, N.B.; Gallardo, F.E. Parasitóides da subfamília Eucoilinae (Hymenoptera: Cynipoidea: Figitidae) coletados em um remanescente de mata de cerrado em Itumbiara, GO. Arquivos do Instituto Biológico, São Paulo, v.68, n.1, p.65-67, 2001. Disponível em: 〈http://www. biologico.sp.gov.br/arquivos/V68_1/12.pdf>. Acesso em: 20 jan. 2006.

Marchiori, C.H.; Pereira, L.A.; Borges, V.R.; Ribeiro, L.C.S.; Silva Filho, O.M.;Diaz, N.B.;G allardo, F.Parasitóides da subfamília Eucoilinae (Hymenoptera: Figitidae) coletados em armadilhas de bacias amarelas e armadilhas Malaise em Araporã, Minas Gerais e Itumbiara, Goiás. Arquivos do Instituto Biológico, São Paulo, v.70, n.2, p.207-209, 2003. Disponível em: <http://www. biologico.sp.gov.br/arquivos/V70_2/marchiori3. pdf>. Acesso em: 20 jan. 2006.

Masner, L. Superfamily Platygastroidea. In: Goulet, H.; Huber, J.T. (Eds.). Hymenoptera of the world: an identification guide to families. Ontario: Agriculture Canada Publication, 1993. 668p. Chapter 14.

PARRA, J.R.P.; Botelho, P.S.M.; CorRÊA-FerReIRA, B.S.; Bento, J.M.S. Controle biológico: terminologia. In: (Eds.). Controle biológico no Brasil: parasitóides e predadores. São Paulo: Manole, 2002. 635p. Cap.1.

Perioto, N.W. Perfil da fauna de Hymenoptera Parasitica, incluindo Chrysidoidea, do cerrado da Fazenda Canchim (EMBRAPA, São Carlos, SP). 1991. 70p. Dissertação (Mestrado) - Universidade Federal de São Carlos, São Carlos, 1991.

Perioto, N.W.; LaRa, R.I.R.; Santos, J.C.C. dos; Selegatto, A. Himenópteros parasitóides (Insecta, Hymenoptera) coletados na cultura de algodão (Gossypium hirsutun L.) (Malvaceae), no município de Ribeirão Preto, SP, Brasil. Revista Brasileira de Entomologia, v.46, n.2, p.165168, 2002a.

Perioto, N.W.; Lara, R.I.R.; Santos, J.C.C. dos; Silva, T.C. Himenópteros parasitóides (Insecta, Hymenoptera) coletados na cultura de soja (Glycine max (L.) Merril) (Fabaceae), no município de Nuporanga, SP, Brasil. Revista Brasileira de Entomologia, v.46, n.2, p.185-187, $2002 b$.

Perioto, N.W.; LaRa, R.I.R. Himenópteros parasitóides (Insecta : Hymenoptera) da Mata Atlântica. I. Parque Estadual da Serra do Mar, Ubatuba, SP, Brasil. Arquivos do Instituto Biológico, São Paulo, v.70, n.4, p.441-445, 2003. Disponível em: \ttp://www.biologico.sp. gov.br/arquivos/V70_4 / perioto.pdf $>$. Acesso em: 20 jan. 2006.

Perioto, N.W.; Lara, R.I.R.; Selegatto, A.; Luciano, E.S. Himenópteros parasitóides (Insecta, Hymenoptera) coletados na cultura de café Coffeaarabica L. (Rubiaceae) em Ribeirão Preto, SP, Brasil. Arquivos do Instituto Biológico, São Paulo, v.71, n.1, p.41-44, 2004. Disponível em: \http://www.biologico.sp.gov.br/arquivos/v71_1/perioto2.pdf>. Acesso em: 20 jan. 2006. 
Perioto, N.W.; Lara, R.I.R.; Selegatto, A. Himenópteros parasitóides da Mata Atlântica.II. Núcleo Grajaúna, Rio Verde da Estação Ecológica Juréia-Itatins, Iguape, SP, Brasil. Arquivos do Instituto Biológico, São Paulo, v.72,n.1, p.81-85, 2005. Disponívelem: <http://www. biologico.sp.gov.br/arquivos/V72_1/perioto2. PDF>. Acesso em: 20 jan. 2006.

Quicke, D.L.J. Parasitic wasps. London: Chapman \& Hall, 1997. 470p.

Restello, R.M.; Penteado-Dias, A.M. Diversidade dos Braconidae (Hymenoptera) da Unidade de Conservação Teixeira Soares, Marcelino Ramos, RS, com ênfase nos Microgastrinae. Revista Brasileira de Entomologia, n.50, v.1, p.80-84, 2006.

Scatolini, D.; Penteado-Dias, A.M. Análise faunística de Braconidae (Hymenoptera) em três áreas de mata nativa do Estado do Paraná, Brasil. Revista Brasileira de Entomologia, v.47, n.2, p.187-195, 2003.
ShAPIRO. B.A.; Rckering, J. Rainfall and parasitic wasp (Hymenoptera: Ichneumonoidea) activity in succesional forest stages at Barro Colorado Nature Monument, Panama, and La Selva BiologicalStation, Costa Rica. Agricultural and Forest Entomology, v.2, p.39-47, 2000.

Silva, C.G., Marchiori, C.H., Torres, L.C. Eucoilinae (Hymenoptera: Figitidae) coletados em Lavras, Minas Gerais. Arquivos do Instituto Biológico, São Paulo, v.70,n.4,p.425-427,2003. Disponívelem:< http://www. biologico.sp.gov.br/arquivos/V70_4/silva1.pdf $>$. Acesso em: 20 jan. 2006.

Recebido em 27/4/06

Aceito em 14/10/06 